\title{
Sustained Neural Activity Patterns during Working Memory in the Human Medial Temporal Lobe
}

\author{
Nikolai Axmacher, ${ }^{1,2}$ Florian Mormann, ${ }^{1}$ Guillén Fernández, ${ }^{3}$ Michael X Cohen, ${ }^{1,2,4}$ Christian E. Elger, ${ }^{1,2}$ and \\ Juergen Fell ${ }^{1}$ \\ ${ }^{1}$ Department of Epileptology, University of Bonn, 53105 Bonn, Germany, ${ }^{2}$ Life and Brain GmbH, 53127 Bonn, Germany, ${ }^{3}$ F. C. Donders Center for Cognitive \\ Neuroimaging and Department of Neurology, Radboud University Nijmegen, 6500 HB Nijmegen, The Netherlands, and ${ }^{4}$ Department of Psychology, \\ University of California, Davis, Davis, California 95616
}

In contrast to classical findings that the medial temporal lobe (MTL) specifically underlies long-term memory, previous data suggest that MTL structures may also contribute to working memory (WM). However, the neural mechanisms by which the MTL supports WM have remained unknown. Here, we exploit intracranial EEG to identify WM-specific sustained activity patterns with the highest temporal and spatial resolution currently available in humans. Using a serial Sternberg paradigm, we found a positive shift of the direct current (DC) potential and a long-lasting decrease in MTL gamma-band activity during maintenance of a single item, reflective of a sustained reduction in neural activity. Maintenance of an increasing number of items elicited an incrementally negative shift of the DC potential and an increase in MTL gamma-band activity. In addition, the paradigm was conducted in healthy control subjects using functional magnetic resonance imaging. This confirmed that our results were not caused by pathological processes within the MTL, and that this region was indeed specifically activated during the task. Our results thus provide direct evidence for sustained neural activity patterns during working memory maintenance in the MTL, and show that these patterns depend on WM load.

Key words: working memory; intracranial EEG; fMRI; medial temporal lobe; hippocampus; DC shifts; gamma band activity

\section{Introduction}

The first historical attempts to localize specific memory systems were based on lesion studies in patients and animals. The results were generally interpreted as indicating that medial temporal lobe (MTL) regions support declarative long-term memory (LTM) encoding and retrieval (Scoville and Milner, 1957; Penfield and Milner, 1958), but are not required for working memory (WM) maintenance over a short time interval (Cave and Squire, 1992). Previous findings, however, questioned this seemingly clear distinction between a LTM system in the MTL and an extrahippocampal (e.g., prefrontal) WM system (Ranganath and Blumenfeld, 2005). Some functional magnetic resonance imaging (fMRI) and clinical studies showed evidence for a contribution of the MTL to WM tasks even if only a single item was maintained (Ranganath and D'Esposito, 2001; Schon et al., 2004; Nichols et al., 2006), whereas others found an involvement only in maintenance of multiple items (Aggleton et al., 1992) or conjunctive features (Hannula et al., 2006; Olson et al., 2006; Piekema et al., 2006). These latter findings are consistent with the views that the MTL is recruited whenever relations between items

Received 0ct. 10, 2006; revised April 14, 2007; accepted May 3, 2007.

This work was supported by Volkswagen Foundation Grant I/79878. We thank Pascal Fries and Ole Jensen for their contribution in designing the experiment, Peter Klaver for help with the implementation, Vera Dinkelacker for providing the stimuli, Christian Bien for support with the clinical management of the patients, and Sven Haupt and Nikolaus Maier for fruitful discussions and valuable comments on a previous draft of this manuscript.

Correspondence should be addressed to Dr. Nikolai Axmacher, Department of Epileptology, University of Bonn, Sigmund-Freud-Strasse 25, D-53105 Bonn, Germany. E-mail: nikolai.axmacher@ukb.uni-bonn.de.

DOI:10.1523/JNEUROSCI.0962-07.2007

Copyright $\odot 2007$ Society for Neuroscience $\quad$ 0270-6474/07/277807-10\$15.00/0 are encoded (Cohen and Eichenbaum, 1993; Eichenbaum, 2004) or when sequences of items are processed (Eichenbaum et al., 1999; Henke et al., 1999; Schendan et al., 2003; Jensen and Lisman, 2005; Kumaran and Maguire, 2006). However, even with a task designed to measure WM processes, a contribution of simultaneous LTM processes is almost impossible to exclude. Thus, activation of the MTL during WM tasks might rather reflect recruitment of LTM processes. Furthermore, neither the relational memory theory nor the sequence encoding theory of the MTL explain why some studies found an MTL contribution even to WM maintenance of single items.

In this situation, we used intracranial EEG (iEEG) recordings in combination with $\mathrm{PMRI}$ to directly investigate the neural processes within the MTL during maintenance of a single and multiple items. IEEG recordings in epilepsy patients provide an important link between electrophysiological recordings in animals and neuroimaging studies performed in human subjects and allow to test specific hypotheses from animal studies: First, sustained modifications of activity were observed while animals maintained items in WM (Suzuki et al., 1997; Young et al., 1997), suggesting an effect on the direct current (DC) component of the medial temporal EEG during WM maintenance in humans. Second, in vitro data indicate a stepwise increase of neural activity in the MTL after repeated electrical stimulations (Egorov et al., 2002), which might translate into activity increases when multiple consecutively presented stimuli have to be maintained. Finally, we tested predictions from an influential computer model of WM processes that proposes that MTL gamma-band activity 
$(\sim 30-100 \mathrm{~Hz})$ increases with the number of items being held in WM (Lisman and Idiart, 1995; Jensen and Lisman, 2005). In addition to the iEEG recordings, we used fMRI in healthy control subjects to rule out that our iEEG results were contaminated by MTL pathology or by unspecific activation of extensive brain regions.

\section{Materials and Methods Subjects}

$i E E G$. Eleven patients with pharmacoresistant temporal lobe epilepsy (three women; mean age \pm SD, 37.2 \pm 12.1 years; handedness: nine right, two left) participated in the study. In seven patients, unilateral hippocampal sclerosis was confirmed histologically. From the others, one had a unilateral isolated amygdala lesion, two others had no apparent MRI lesions, and one had a unilaterally accentuated limbic pathology. Recordings were performed from 2004 to 2005 at the Department of Epileptology, University of Bonn, Germany. Ten patients had bilateral hippocampal depth electrodes, and one had a single electrode in the right hippocampus and an extrahippocampal (temporo-occipital) seizure onset zone. No seizure occurred within $24 \mathrm{~h}$ before the experiment.

Additional experiments were performed by six other epilepsy patients (one woman; mean age $\pm \mathrm{SD}, 42.7 \pm 3.3$ years; handedness: five right, one ambidexter) (see supplemental material, available at www. jneurosci.org). Three of them (who performed the Sternberg experiment with a subsequent long-term recognition task) had bilateral MTL depth electrodes and a unilateral seizure onset zone; the other three patients had electrodes in the primary motor cortex (as ascertained by electrical mapping, i.e., function loss caused by electrical stimulation).

fMRI. Twenty-three healthy subjects ( 12 women; mean age $\pm \mathrm{SD}$, $30.0 \pm 10.4$ years; handedness: 22 right, 1 left) participated in the study. They were recruited from the University of Bonn as well as via newspaper.

Complementary behavioral study. Eighteen healthy subjects (five women; mean age $\pm \mathrm{SD}, 27.0 \pm 6.5$ years) recruited from the University of Bonn participated in the study.

The EEG, the fMRI, and the behavioral study were approved by the local medical ethics committee, and all patients and subjects gave written informed consent.

\section{Experimental paradigm}

Overview. Our modified Sternberg WM paradigm with serial presentation of items allows for parametric modulation of the WM load (i.e., the number of items that have to be maintained over a short interval). Subjects had to memorize one, two, or four black and white photographs of unknown male and female faces (total of 126 male and 126 female faces) that had been rated previously by a large group of subjects as neutral with respect to facial expression. Trial timings differed slightly for the iEEG and fMRI experiments to account for different temporal signal characteristics (described below). The experiment was run in a single block containing a total of 108 trials (i.e., 36 in each condition; one, two, or four faces). This resulted in a total of 306 presented faces; in addition to the faces shown as items in the trials with one, two, and four faces $(36 \times 1+$ $36 \times 2+36 \times 4=252$ faces), 54 additional faces were used as probes in the nonmatch trials. The different trials types were presented in random order. Subjects were instructed to complete the task as quickly and accurately as possible. Figure 1 provides an overview of the paradigm in the two conditions. Only trials with a correct response were taken into account for EEG and fMRI analyses.

$i E E G$. Each picture was presented using ERTS software (BeriSoft, Frankfurt, Germany) in the center of a computer screen for $500 \mathrm{~ms}$ with a randomized interstimulus interval with a mean of $1500 \mathrm{~ms}$, and a range of 1300-1700 ms ("encoding" phase). Afterward, patients had to maintain the faces in WM for $3000 \mathrm{~ms}$ ("maintenance" phase). Subsequently, patients were presented a probe for $500 \mathrm{~ms}$ and had to decide whether it matched one of the faces seen during that trial's encoding phase ("target") or not ("nontarget"). Half of the trials were target and half were nontarget trials, respectively. All pictures were presented at a size of $6.9^{\circ}$ (horizontal) and $10.4^{\circ}$ (vertical) visual angle. The length of the intertrial interval was $5000 \mathrm{~ms}$. Faces were shown only within one trial and were not reused during the experiment. Patients indicated their decision by pressing one of two buttons of a computer mouse in their dominant hand. A total of 306 faces was presented to each patient in one block containing 108 trials; the overall duration of the experiment was $\sim 20$ min. During the experiment, we recorded continuous EEG from the depth electrodes as well as from bilateral mastoid electrodes.

$f M R I$. The fMRI experiment was identical to the iEEG experiment with the following exceptions. Stimulus presentation time during the encoding phase was $800 \mathrm{~ms}$ with a constant interstimulus interval of $1000 \mathrm{~ms}$. The maintenance phase was extended to a mean of $8000 \mathrm{~ms}$ (range, $6000-10,000 \mathrm{~ms}$ ) so the blood oxygen level-dependent (BOLD) response during this period could be modeled more accurately (Zarahn et al., 1997; Postle et al., 2000; Ranganath and D'Esposito, 2001; Schon et al., 2004). The test face was presented for $1500 \mathrm{~ms}$, and the duration of the intertrial interval was $4000 \mathrm{~ms}$. Pictures were presented at a size of $10.1^{\circ}$ (horizontal) and $15.1^{\circ}$ (vertical) visual angle. To ensure equal length of the encoding phase and to match perceptual parameters between trials with one, two and four faces, we always presented a total of four items that were either faces or scrambled faces (four faces or two faces and two scrambled faces or one face and three scrambled faces). Scrambled faces were generated using the "pixel transformation effect" feature of Jasc Paint Shop Pro (Corel, Ottawa, Canada), which replaces the original color values in nonoverlapping adjacent windows with the average color values in these windows (and thus basically acts as a low-pass filter). In trials containing scrambled faces, these were presented at random positions within the trials. Stimuli were presented through goggles (NordicNeuroLab, Bergen, Norway) using E-Prime software (Psychology Software Tools, Pittsburgh, PA). Again, the experiment contained 108 trials and lasted $\sim 42 \mathrm{~min}$, including six randomly interspersed pauses of $30 \mathrm{~s}$ duration each that were used as a baseline.

\section{Recording}

iEEG. Multicontact depth electrodes were inserted for diagnostic purposes using a computed tomography-based stereotactic insertion technique (Van Roost et al., 1998). The location of electrode contacts was ascertained by MRI in each patient and was classified as either hippocampal, rhinal, or other. Because our methods cannot clearly separate perirhinal and entorhinal generators, we use the term rhinal cortex without indicating an integrated rhinal processing stage. On average, patients had $2.2 \pm 0.6$ rhinal and $5.4 \pm 1.9$ hippocampal contacts (mean \pm SD). Depth EEG was referenced to linked mastoids, recorded at a sampling rate of $1000 \mathrm{~Hz}$, and bandpass filtered $[0.01 \mathrm{~Hz}(6 \mathrm{~dB} /$ octave $)$ to $300 \mathrm{~Hz}$ (12 dB/octave)]. In the 10 patients with bilateral depth electrodes, as well as in the three additional patients who performed the Sternberg experiment with the subsequent long-term memory recognition task, data were recorded bilaterally, but only data from the hemisphere contralateral to the seizure onset zone were used for data analysis. In the one patient with a unilateral depth electrode, data from that electrode, which was distant from the temporo-occipital seizure onset zone, were used. In the three patients with electrodes in the primary motor cortex, data from all electrodes within the primary motor cortex $(8,9$, and 11 electrodes in the three patients) were averaged.

fMRI. Sixteen axial slices were collected at 1.5T (Avanto; Siemens, Erlangen, Germany). We collected $780 \mathrm{~T} 2^{\star}$-weighted, gradient echo echo-planar imaging scans, including five initial scans that were discarded to achieve steady-state magnetization (slice thickness, $3 \mathrm{~mm}$; interslice gap, $0.3 \mathrm{~mm}$; matrix size, $64 \times 64$; field of view, $192 \mathrm{~mm}$; echo time, $40 \mathrm{~ms}$; repetition time, $3200 \mathrm{~ms}$ ). Thereafter, we acquired a sagittal T1-weighted magnetization-prepared rapid-acquisition gradient echo sequence for each subject for anatomical localization (number of slices, 160; slice thickness, $1 \mathrm{~mm}$; interslice gap, $0.5 \mathrm{~mm}$; matrix size, $256 \times 256$; field of view, $256 \mathrm{~mm}$; echo time, $3.93 \mathrm{~ms}$; repetition time: $1660 \mathrm{~ms}$ ).

\section{Analyses}

Event-related potential data. EEG trials were visually inspected for artifacts (e.g., epileptiform spikes), and $12.6 \%$ of all trials were excluded from analysis. From the contralateral (nonfocal) electrode in each 
patient, data from all hippocampal and all rhinal contacts as determined from postimplantation MRI were averaged, yielding one accumulated rhinal and one hippocampal data set for each patient. This was done to avoid any bias introduced by the selection of rhinal or hippocampal electrode contacts; similar results were obtained when only the hippocampal and the rhinal contact with the maximal slope or the maximal absolute slope of the DC potential were analyzed. We analyzed activity both during the maintenance phase (in trials with one, two, and four items), and during presentation of subsequent stimuli in the encoding phase (only in trials with four items). Long-lasting (DC) shifts of the EEG were analyzed by calculating both the average (across subsequent windows of $1000 \mathrm{~ms}$ length) and the slope (windows, 0-1000, 0-2000, and $0-3000 \mathrm{~ms}$ ). The slope was calculated as the fit of a linear regression line to the event-related potentials (triggered to the onset of the last face in each trial to avoid stimulus-related event-related potentials in the baseline period) averaged across trials. Statistical analyses of both DC slopes and DC averages were performed separately for the different windows because of the high correlation of the data in these windows. Data were baseline corrected to an interval between -200 and $0 \mathrm{~ms}$ before the last stimulus in each set before the beginning of the maintenance phase (i.e., before the onset of the first stimulus in trials with one face, the second stimulus in trials with two faces, and the fourth stimulus in trials with four faces). For the analysis of DC potentials during the encoding phase in trials with four faces, the baseline was accordingly chosen as the interval between -200 and $0 \mathrm{~ms}$ before the onset of the first, second, third, or fourth stimulus. Data were analyzed using the EEGLAB package created by A. Delorme and S. Makeig (Delorme and Makeig, 2004) run with Matlab (Mathworks, Natick, MA).

Power changes. In addition to time-domain analyses, we conducted frequency-based analyses to assess whether and how localized bursts of activity in specific frequency bands reflected task demands. EEG trials were filtered in the frequency range from 2 to $100 \mathrm{~Hz}$ ( $2 \mathrm{~Hz}$ steps) by continuous wavelet transforms implementing Morlet wavelets of five cycles length (Daubechies, 1990; Torrence and Compo, 1998). The filtered signals result from the time convolution of original signals and the complex wavelet function. To avoid edge effects, the trials entering the wavelet transform were segmented from -2.4 to $5.2 \mathrm{~s}$ with respect to presentation of the last stimulus in each trial (i.e., the first stimulus in trials with one face, the second stimulus in trials with two faces, and the fourth stimulus in trials with four faces). An interval of $2.2 \mathrm{~s}$ at the beginning and the end of the trials was afterward discarded. For statistical analyses, power values were averaged for nonoverlapping successive time windows of $1000 \mathrm{~ms}$ duration from 0 to $3000 \mathrm{~ms}$ after the onset of the last stimulus in each trial. Afterward, power values were normalized with respect to the prestimulus time window from -200 to $0 \mathrm{~ms}$ separately for each subject and each filter frequency. For graphical depiction, power values were normalized to the prestimulus time window and then transformed into decibel scale $\left(10 \times \log _{10}\right)$. Because of the intrinsic logarithmic frequency scaling of the wavelet decomposition, higher frequencies are not statistically independent if sampled too closely on an equidistant frequency scale. Therefore, the EEG was selectively analyzed in the gamma frequency range in the following spectral bands: $\gamma_{1}, 20-34 \mathrm{~Hz}$; $\gamma_{2}, 36-50 \mathrm{~Hz} ; \gamma_{3}, 52-66 \mathrm{~Hz} ; \gamma_{4}, 68-82 \mathrm{~Hz} ; \gamma_{5}, 84-98 \mathrm{~Hz}$. $p$ values in the ANOVAs were Huynh-Feldt corrected for inhomogeneities of covariance when necessary (Huynh and Feldt, 1976).

fMRI. MRIs were processed in SPM2 (www.fil.ion.ucl.ac.uk/spm/) using standard preprocessing steps including realignment, unwarping, normalization, and smoothing with an $8 \mathrm{~mm}$ Gaussian kernel. Preprocessed data were fitted by the convolution of multiple regressors with a canonical hemodynamic response function to obtain parameter estimates for each condition covariate. The following set of regressors was used: three regressors for the encoding phase in trials with one, two, and four items; three regressors for the maintenance phase in trials with one, two, and four items; one regressor for the retrieval phase (independent of memory load); one regressor for the pause periods; and one regressor for the collapsed encoding and maintenance phase in trials with incorrect responses. Then, the following contrasts were defined: first, memory load-independent activation during encoding compared with baseline (contrast parameters, $+1,+1,+1$ for the encoding regressors with

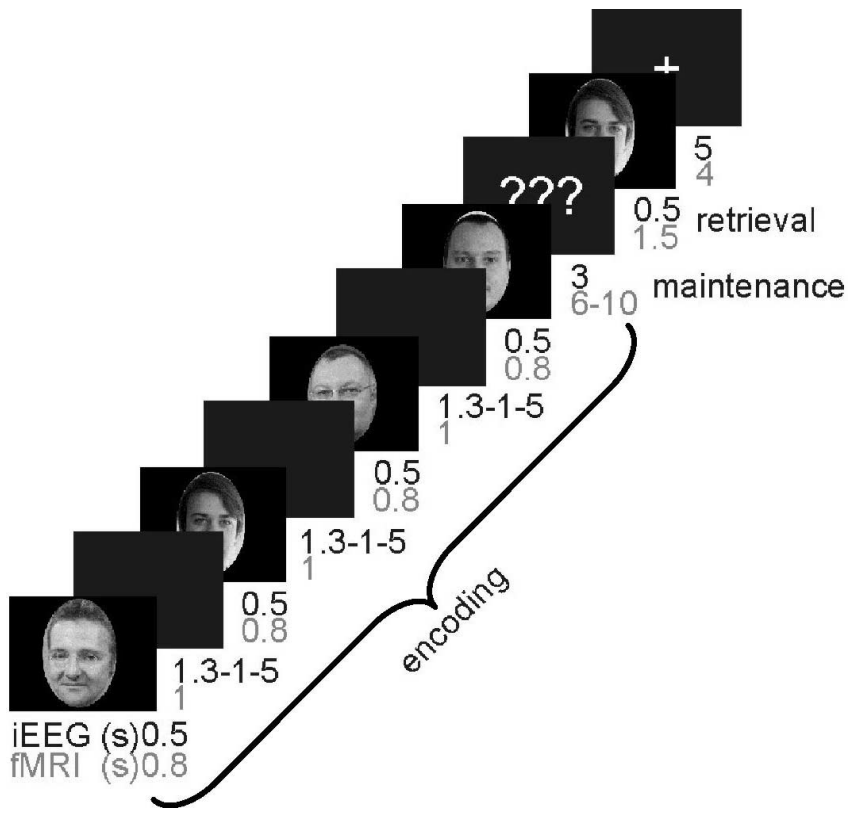

Figure 1. Overview of the paradigm.

different memory load of one, two, and four items, and -3 for the pause, which we used as baseline); second, memory load-independent activation during maintenance compared with baseline (contrast parameters, $+1,+1,+1$ for the maintenance regressors with different memory load of one, two, and four items, and -3 for the pause); third, memory loaddependent activation during encoding $(-1,0,+1$ for the encoding regressors with different memory load of one, two, and four items); and fourth, memory load-dependent activation during maintenance $(-1,0$, +1 for the encoding regressors with different memory load of one, two, and four items). To test whether the memory-load dependent activity during maintenance could be attributed to the early or late maintenance phase, we used a separate general linear model with separate regressors for the early (up to $4 \mathrm{~s}$ after the offset of the fourth stimulus in each trial, which could be either a face or a scrambled face) and late maintenance phase. All figures with fMRI results are displayed using neurological convention (left hemisphere on the left side of the figure). To identify significant activations, we used an uncorrected threshold of $p<0.001$ and a minimum cluster size of 10 contiguous voxels. Time courses for each subject were extracted using the Marsbar extension of SPM (Brett et al., 2002).

\section{Results}

\section{Behavioral data (iEEG and fMRI experiments)}

To assess the contribution of the MTL to WM tasks with a varying memory load, we used a serial Sternberg paradigm (Sternberg, 1975 ) in which subjects had to maintain either one, two, or four faces (Fig. 1). This paradigm allowed us to differentiate between an encoding phase, during which stimuli are presented, a maintenance phase, during which stimuli have to be retained in WM, and a retrieval phase. As expected, accuracy decreased with increasing memory load (Fig. 2) (two-way ANOVA; $F_{(2,64)}=6.19$; $p<0.01 ; \varepsilon=0.91$ ) (for numerical values, see supplemental table 1 , available at www.jneurosci.org as supplemental material). The increase in reaction times with memory load was not significant. fMRI subjects performed significantly better than patients in terms of both accuracy (two-way ANOVA; $F_{(1,32)}=16.4 ; p<$ $0.001)$ and reaction times $\left(F_{(1,32)}=8.16 ; p<0.01\right)$. Importantly, no significant interaction between group and memory load was observed, indicating that the dependence of the behavioral outcome on memory load was similar in both groups. 

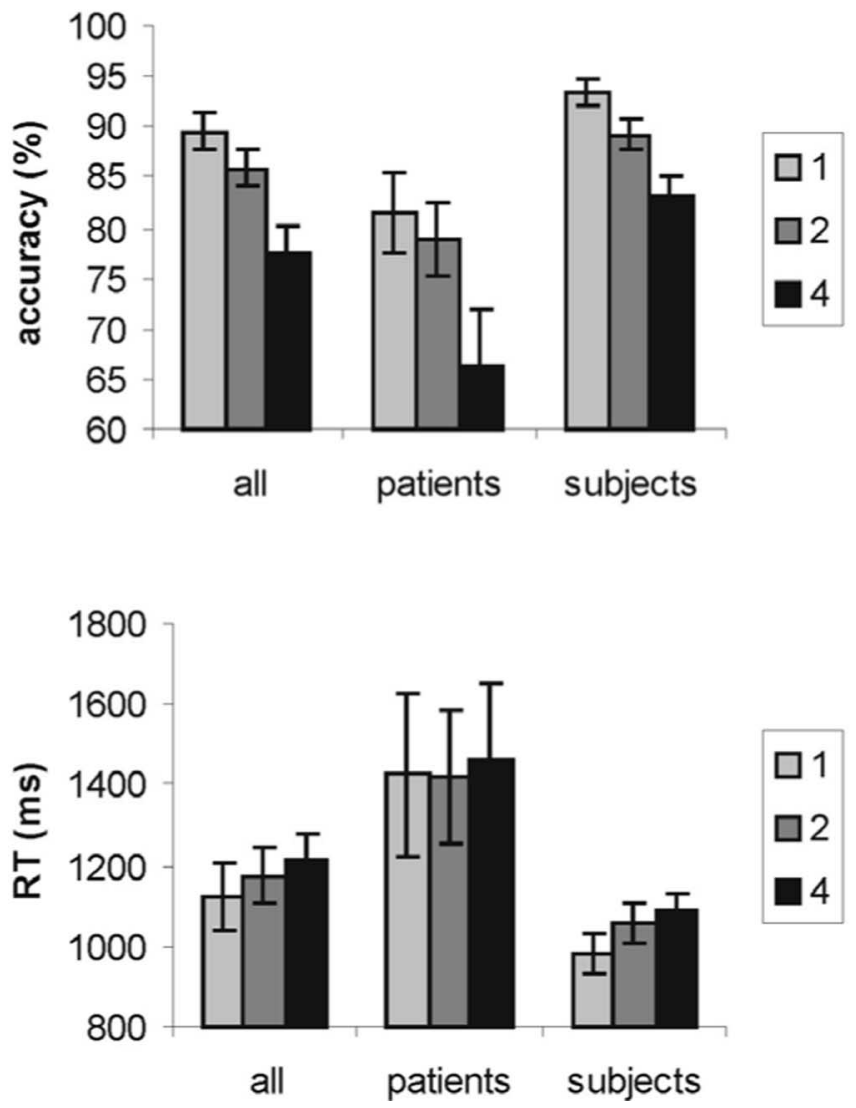

Figure 2. Behavioral results. Top, Accuracy of the entire group of 34 subjects, of the 11 patients, and of the 23 normal subjects as a function of memory load. Bottom, Reaction time. Error bars indicate SEM.

\section{EEG results: event related potentials}

We recorded iEEG from the rhinal cortex and hippocampus of 11 epilepsy patients. The maintenance of items in WM over a short time interval has been shown to be associated with persistent activity changes after stimulus presentation (Fuster and Alexander, 1971; Goldman-Rakic, 1995). We thus investigated whether persistent changes of the EEG could be observed. First, only trials at which a single item is maintained are investigated and changes in activity compared with baseline are analyzed; next, we compared memory load-dependent activity changes during maintenance of multiple items. Finally, we investigated activity during the encoding phase in trials with four items.

\section{Maintenance of a single item}

EEG data were aligned to the onset of the last stimulus, so that the maintenance phase could be directly compared across trials with different memory load. Long-lasting shifts of the EEG were analyzed by calculating both the slope of the DC component of the data (in windows of 1000, 2000, and 3000 ms lengths starting at the presentation of the last stimulus) (see Materials and Methods) and the averaged data (across subsequent windows of 1000 ms length). We found a slow positive shift of the DC potential during maintenance of a single item (Fig. 3, light gray traces). This effect appeared to be present both in the rhinal cortex and in the hippocampus, and it was more pronounced in the latter. The slope of the DC potential was significantly different from baseline in the middle $\left(F_{(1,20)}=7.38 ; p<0.05\right)$ and late $\left(F_{(1,20)}=4.97\right.$; $p<0.05$ ) window, but not in the first one, indicating sustained rather than transient changes of neural activity. There was no interaction with locus (hippocampus vs rhinal cortex). These results indicate that the DC potential shifts toward more positive values both in the hippocampus and in the rhinal cortex when a single item is maintained in WM. The average value of the DC potential showed a trend toward a difference from baseline in the last time window (ANOVA; $F_{(1,20)}=3.90 ; p=0.062$ ), but not during earlier time windows. Again, there was no main effect of locus and no interaction with locus, indicating a similar effect in hippocampus and rhinal cortex. These results demonstrate that the DC potential shifts to more positive values during maintenance of a single item. This effect is significant in later time windows during the maintenance phase and occurs both in the rhinal cortex and in the hippocampus.

\section{Maintenance of multiple items}

Next, we tested whether the shift of the DC potential depends on WM load. Again, we analyzed both the slope of the DC potential and the average voltage values. There were no significant effects of memory load on the DC slope during the short and long window. However, in the middle window, there was a trend toward a main effect of load $\left(F_{(2,20)}=3.28 ; p=0.063 ; \varepsilon=0.934\right)$ as well as a significant interaction between locus and load $\left(F_{(2,20)}=4.73\right.$; $p<0.05 ; \varepsilon=1.095)$. In the hippocampus, an increasing memory load led to a significantly more negative slope of the DC shift $\left(F_{(2,20)}=5.05 ; p<0.05 ; \varepsilon=1.067\right)$, whereas this effect did not reach significance in the rhinal cortex. This suggests that the positive DC shift during maintenance of a single item changed toward a more negative shift when multiple items are maintained in the hippocampus, but not in the rhinal cortex. This memory load-dependent shift of the DC potential is also visible in the average DC potentials: two-way ANOVAs with locus and load as repeated measures revealed a trend for an interaction with locus $\left(F_{(2,20)}=2.52 ; p=0.106 ; \varepsilon=1.199\right)$ and a significant hippocampal effect of load during the middle time window $\left(F_{(2,20)}=3.69\right.$; $p<0.05 ; \varepsilon=0.910)$, but not in the early and late time windows. Thus, the DC potential in the hippocampus, but not in the rhinal cortex shifts toward more negative values during maintenance of an increasing number of items.

\section{Encoding of multiple items (trials with four faces)}

To verify whether the memory-load dependent DC potentials already build up during the presentation of subsequent stimuli, we analyzed mean values and slopes of the DC potential during the $2000 \mathrm{~ms}$ after the onset of items in trials in which four faces were presented (Fig. 4). Trials in which only a single or two faces were presented were not investigated in this analysis. From the maintenance phase, we hypothesized that the slope of the DC potential during a window of $2000 \mathrm{~ms}$ after each stimulus would become more negative for subsequent stimuli, and that the average potential would change toward more negative values during the 1000-2000 ms window as well. Indeed, the slope of the DC potential increased significantly after presentation of subsequent faces both in the rhinal cortex and the hippocampus (two-way ANOVA with intratrial load and locus as repeated measures; $\left.F_{(3,30)}=4.42 ; p<0.05 ; \varepsilon=0.777\right)$. This effect was significant when it was calculated across the entire length of $2000 \mathrm{~ms}$ after stimulus presentation, but not during the first $1000 \mathrm{~ms}$, again pointing toward alterations of sustained, but not transient neural activity. Similarly, the average DC potential showed a trend for a load effect $\left(F_{(3,30)}=2.51 ; p=0.080 ; \varepsilon=0.975\right)$ in both regions during the second, but not during the first $1000 \mathrm{~ms}$ after stimulus presentation. Together, the negative shift of the DC potential during maintenance of multiple items results from a gradual 

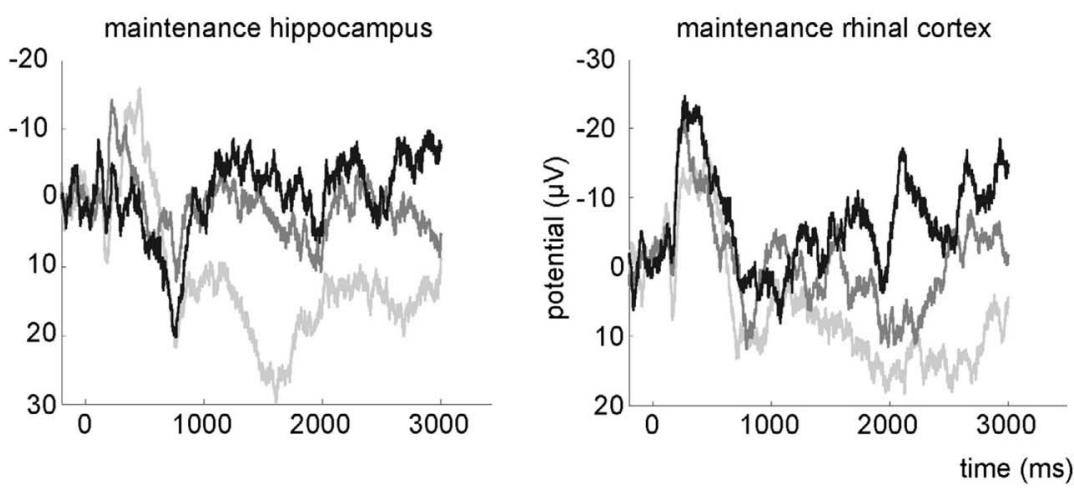

Figure 3. Positive shift of the $D C$ potential during maintenance of a single item turning increasingly negative during maintenance of multiple items. Left column, Averaged hippocampal contacts; right column, averaged rhinal contacts in trials with one (light gray), two (dark gray), and four items (black).
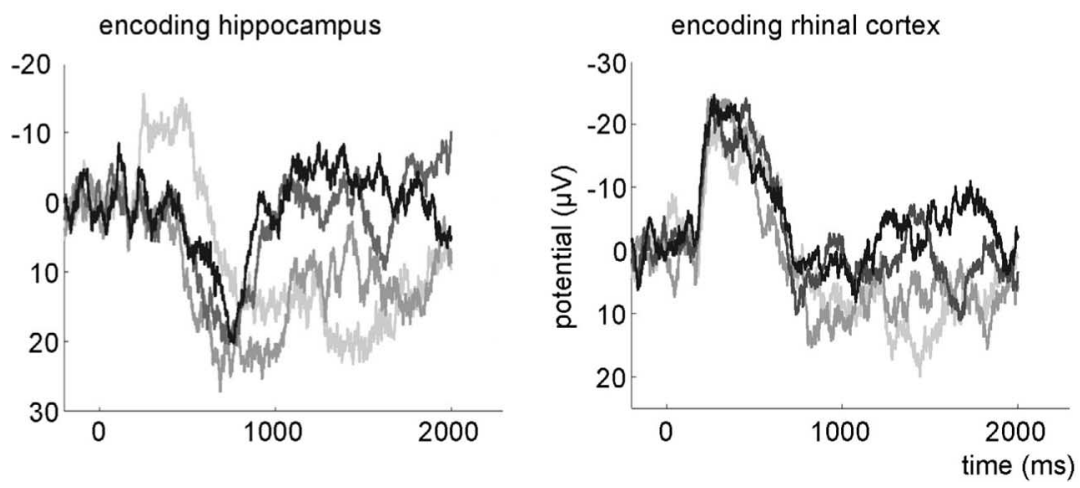

Figure 4. Build-up of the negative shift of the $D C$ potential during encoding of subsequent items in trials with four items. Left column, Averaged hippocampal contacts; right column, averaged rhinal contacts. Darker shades of gray indicate items later in the trial.
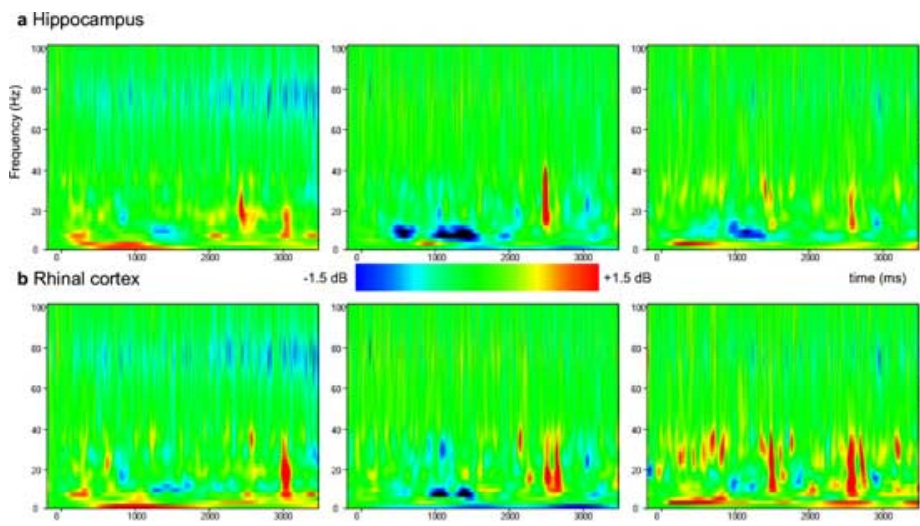

Figure 5. Decreased gamma power during maintenance of a single item and increasing gamma power during maintenance of multiple items. Trials with one item (left), two items (medium), and four items (right column). $\boldsymbol{a}, \boldsymbol{b}$, Power of iEEG from hippocam$\mathrm{pal}(\boldsymbol{a})$ and rhinal $(\boldsymbol{b})$ contacts. The plots on the right display normalized power values in the gamma frequency range $(20-98 \mathrm{~Hz})$ for trials with one item (light gray), two items (dark gray), and four items (black). Error bars indicate SEM.

build-up of a more negative potential during encoding, which is significant both in the hippocampus and in the rhinal cortex.

\section{Specificity of load effects}

It might be argued that the DC shifts reflect solely perceptual processes and are independent of the correct maintenance of a different number of items. To exclude this possibility, we analyzed DC potentials in trials which were incorrectly answered (WM trials). Supplemental Table 2 (available at www.jneurosci.
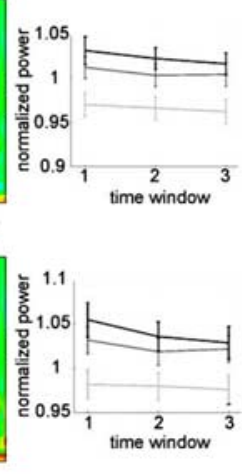

org as supplemental material) provides an overview of the findings in the hippocampus, where the load effect was most pronounced (calculated for an extended group of 14 subjects) (see below). For the WM trials, there was no load-dependent shift of the DC potential neither in the slope of a linear regression line nor in the average values during maintenance. During encoding, there was a trend for an intratrial load effect on average values in the first window; however, the values in this window did not change monotonically with load.

To test whether the load-dependent shifts of the DC potential reflect unspecific effects, we recorded iEEG in an additional group of three patients with subdural grid electrodes in the motor cortex while they performed the same paradigm. These patients were selected because in contrast to various regions in the frontal, temporal, and parietal lobe, primary motor regions are probably not involved in WM processes. We did not observe consistent shifts of the DC potential in these electrodes (supplemental Fig. 1, available at www.jneurosci.org as supplemental material), and there was no significant effect of load neither on the slope nor on the average values.

\section{EEG results: gamma-band activity}

Gamma-band activity is a prominent oscillatory pattern during activated states in the MTL that is related to cellular correlates of WM maintenance (Egorov et al., 2002) (see Discussion). We thus investigated the power of network oscillations in the gamma frequency range $(20-98 \mathrm{~Hz})$ during maintenance of a single item and as a function of memory load during the maintenance phase. We found that gamma power was reduced during maintenance of a single item compared with baseline (Fig. 5). A four-way ANOVA with frequency band (five different bands in the gamma range with $14 \mathrm{~Hz}$ bandwidth each) as the independent variable and subsequent windows of $1000 \mathrm{~ms}$ length and locus as repeated measures was used to statistically test for this decrease of gammaband activity during maintenance of a single item. We found a significant interaction of the difference from baseline power with frequency band, time window, and locus $\left(F_{(2,212)}=3.70 ; p<0.05 ; \varepsilon=\right.$ $0.638)$. In the hippocampus, but not in the rhinal cortex, there was a significant reduction of oscillatory power in the upper gamma frequency range during later time windows; in the middle time window, gamma power was significantly smaller than during baseline in the frequency from 84 to $98 \mathrm{~Hz}$ (one-tailed $t$ test; $p<0.05$ ), whereas in the last time window it was significantly reduced over a larger frequency range from 52 to $98 \mathrm{~Hz}$ (one- 
a

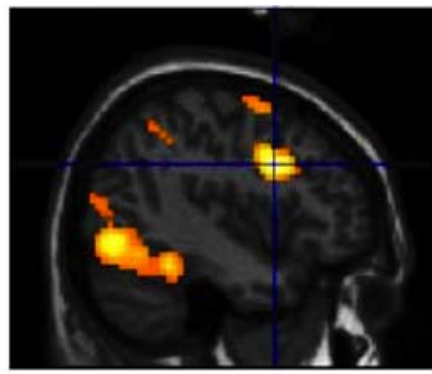

b

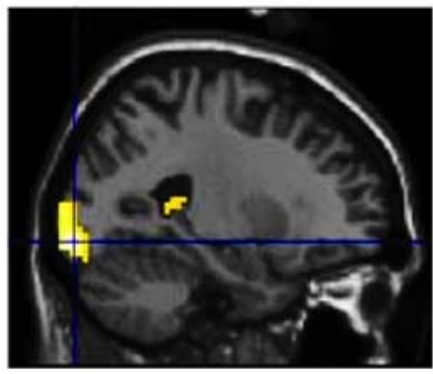

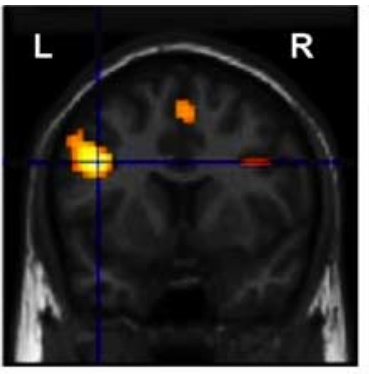

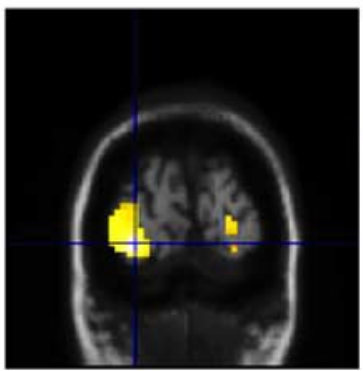

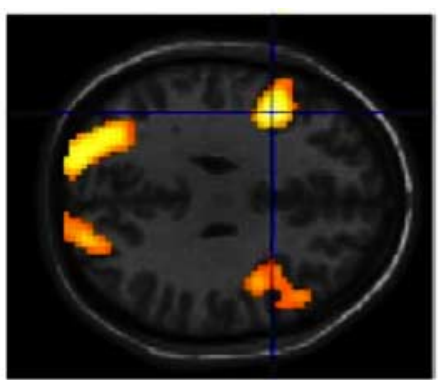
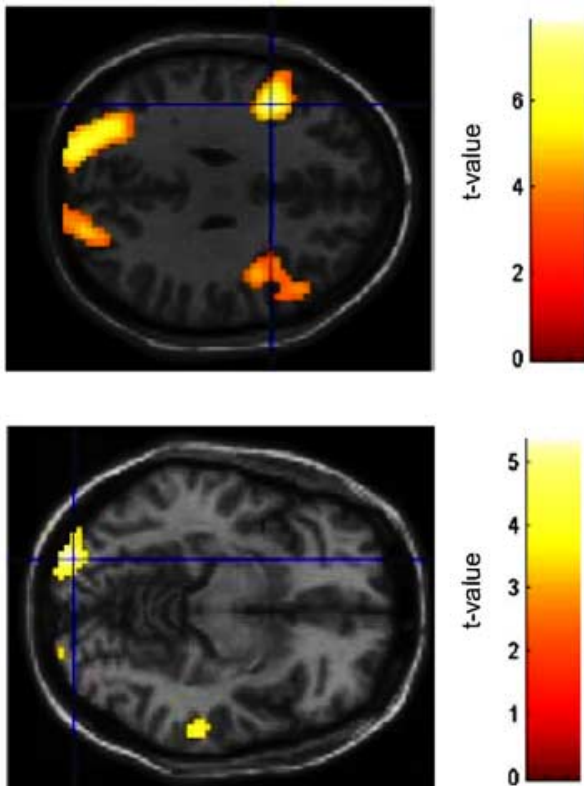

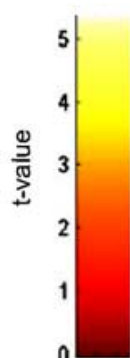

DLPFC
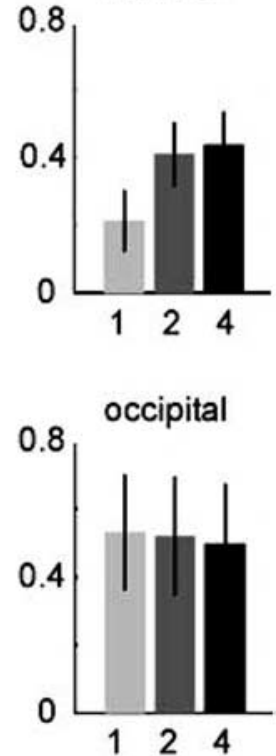

Figure 6. Activation during encoding and maintenance period regardless of memory load. $a$, During encoding, we observed bilateral activation in the prefrontal cortex as well as in higher visual regions. Parameter estimates for different memory loads are shown for the contrast estimates in the most activated voxel in the left middle frontal gyrus (mean \pm SD). $\boldsymbol{b}$, Activation pattern during maintenance compared with rest. Parameter estimates are shown for the contrast estimates in the most activated voxel in the left inferior occipital gyrus (mean \pm SD). In none of these contrasts did we observe an activation of MTL regions.

tailed $t$ test; $p<0.05)$. There was no significant change in the first time window.

Next, we analyzed whether gamma-band activity increased during maintenance of an increasing number of items. We found that, contrary to the memory load effect on the DC potential, which was more pronounced in the hippocampus, gamma power increased significantly only in the rhinal cortex: a four-way ANOVA with frequency band as independent variable and locus, memory load, and subsequent time windows of $1000 \mathrm{~ms}$ length as repeated measures showed a trend toward a main effect of load $\left(F_{(2,106)}=2.60 ; p=0.080 ; \varepsilon=0.991\right)$, a significant interaction of locus and load $\left(F_{(2,106)}=6.05 ; p<0.01 ; \varepsilon=0.964\right)$, and a significant load effect in the rhinal cortex $\left(F_{(2,106)}=4.01 ; p<\right.$ 0.05; $\varepsilon=0.973$ ). No significant load effect was observed in the hippocampus.

Finally, we tested whether the results were specific to the gamma frequency band. We calculated a four-way ANOVA of power values in the $\delta(2 \mathrm{~Hz})$, theta $(4-8 \mathrm{~Hz}), \alpha(10-12 \mathrm{~Hz})$, and $\beta(14-18 \mathrm{~Hz}$ ) frequency bands (again with load, locus, and time window as repeated measures and band as the independent variable). There was no main effect of load and no load by band interaction. In addition, there was no effect of load in any of the frequency bands when analyzed separately.

\section{fMRI results}

To confirm that our iEEG findings were not contaminated by MTL pathology in the epileptic patients and that they do not reflect unspecific activity changes of extensive brain regions, we conducted the same paradigm in 23 healthy control subjects using fMRI. We first attempted to localize brain regions that exhibited task-related activation regardless of memory load and therefore compared activation during the encoding (stimuluspresentation) and maintenance phases relative to baseline. As a baseline we defined pause periods of $30 \mathrm{~s}$ duration instead of intertrial intervals to avoid contamination with BOLD responses from stimulus presentation. These results are displayed in Figure
6. All significantly activated regions of the different contrasts are listed in Table 1. Most importantly, no significant activations in the MTL were observed in these contrasts, in which activity was averaged across trials with different memory load.

Our iEEG findings showed that DC potentials within the MTL shifted toward more negative values with increasing WM load. We therefore separately analyzed WM processes during trials with different numbers of items during encoding and maintenance (Fig. 7). We found increased activation in left MTL regions during both encoding and maintenance as a function of increased WM load. During encoding, we observed activations in the left hippocampus $\left(t_{(22)}=4.16\right.$; MNI coordinates, $\left.-18,-30,-3\right)$, as well as bilateral activations in visual areas in the lateral occipital gyrus (left: $t_{(22)}=8.36$; MNI coordinates, $-42,-84$, -9 ; right: $t_{(22)}=6.94$, MNI coordinates, 39, $\left.-84,-6\right)$, and in the left middle $\left(t_{(22)}=4.79\right.$; MNI coordinates, $\left.-42,12,27\right)$ and right inferior frontal gyrus $\left(t_{(22)}=4.72 ;\right.$ MNI coordinates, 54, 27, 18). The region in the left middle frontal gyrus is probably the dorsolateral prefrontal cortex. During maintenance, we observed activation of the left hippocampus as well $\left(t_{(22)}=4.29\right.$; MNI coordinates, $-24,-18,-12$ ) (a time course of activation in this cluster is depicted in the supplemental Fig. 3, available at www.jneurosci. org as supplemental material). Interestingly, as seen in the right column of Figure 7, the parameter estimates for the medial temporal contrast estimates both of the encoding and the maintenance regressors are negative for a memory load of a single item, close to zero for two items, and positive for four items. This difference explains why activations in these regions were not observed when simply comparing all activations against the baseline condition. As described in the next section, this change from hippocampal deactivation to activation with increasing memory load closely mirrors our iEEG results and indicates a specific involvement of the MTL in WM of multiple items.

Previous work has suggested that activity during the delay period of a WM paradigm can be most accurately assessed by convolving the hemodynamic response function with modeled 


\begin{tabular}{|c|c|c|c|c|c|c|}
\hline \multirow[b]{2}{*}{ Region } & \multirow[b]{2}{*}{$\mathrm{L} / \mathrm{R}$} & \multirow[b]{2}{*}{ BA } & \multicolumn{3}{|c|}{ MNI coordinates } & \multirow[b]{2}{*}{ tscore } \\
\hline & & & $x$ & $y$ & $z$ & \\
\hline \multicolumn{7}{|l|}{ Encoding $>$ pause } \\
\hline Middle frontal gyrus & $\mathrm{L}$ & 9 & -42 & 9 & 30 & 7.96 \\
\hline Cuneus & $\mathrm{L}$ & 18 & -12 & -96 & 15 & 6.86 \\
\hline Middle frontal gyrus & $\mathrm{R}$ & 6 & 42 & 0 & 57 & 5.35 \\
\hline Medial superior frontal gyrus & $\mathrm{L}$ & 8 & 0 & 18 & 51 & 5.30 \\
\hline Middle frontal gyrus & $\mathrm{L}$ & 6 & -45 & 3 & 57 & 4.27 \\
\hline Superior parietal gyrus & $\mathrm{R}$ & 7 & 24 & -66 & 36 & 4.22 \\
\hline Lateral occipital gyrus & $\mathrm{R}$ & 19 & 36 & -81 & 3 & 3.90 \\
\hline Insular cortex & $\mathrm{R}$ & 45 & 36 & 27 & 6 & 3.79 \\
\hline \multicolumn{7}{|l|}{ Maintenance $>$ pause } \\
\hline Inferior occipital gyrus & $\mathrm{L}$ & 18 & -24 & -93 & -9 & 5.41 \\
\hline Posterior cingulate gyrus & $\mathrm{R}$ & & 15 & -39 & 12 & 5.23 \\
\hline Posterior cingulate gyrus & $\mathrm{L}$ & & -18 & -36 & 12 & 5.04 \\
\hline Fusiform gyrus & $\mathrm{L}$ & 19 & -48 & -66 & -21 & 4.60 \\
\hline Middle temporal gyrus & $\mathrm{R}$ & 21 & 63 & -30 & -9 & 4.16 \\
\hline Medial superior frontal gyrus & $\mathrm{R}$ & 6 & 3 & 9 & 54 & 4.09 \\
\hline Middle temporal gyrus & $\mathrm{R}$ & 20 & 57 & -39 & -15 & 3.76 \\
\hline \multicolumn{7}{|l|}{ Memory load: encoding } \\
\hline Lateral occipital gyrus & $\mathrm{L}$ & 18 & -42 & -84 & -9 & 8.36 \\
\hline Lateral occipital gyrus & $\mathrm{R}$ & 19 & 39 & -84 & -6 & 6.94 \\
\hline Middle frontal gyrus & $\mathrm{L}$ & 9 & -42 & 12 & 27 & 4.79 \\
\hline Superior colliculus & $\mathrm{L}$ & & -6 & -27 & -12 & 4.75 \\
\hline Inferior frontal gyrus & $\mathrm{R}$ & 46 & 54 & 27 & 18 & 4.72 \\
\hline Superior occipital gyrus & $\mathrm{R}$ & 7 & 27 & -72 & 33 & 4.48 \\
\hline Superior occipital gyrus & $\mathrm{L}$ & 19 & -30 & -72 & 33 & 4.46 \\
\hline Inferior frontal gyrus & $\mathrm{R}$ & 45 & 57 & 21 & 6 & 4.16 \\
\hline Hippocampus & $\mathrm{L}$ & & -18 & -30 & -3 & 4.16 \\
\hline Medial superior frontal gyrus & $\mathrm{R}$ & 32 & 9 & 21 & 48 & 3.80 \\
\hline Inferior frontal gyrus & $\mathrm{R}$ & 9 & 48 & 15 & 30 & 3.76 \\
\hline \multicolumn{7}{|l|}{ Memory load: maintenance } \\
\hline Hippocampus & $\mathrm{L}$ & 28 & -24 & -18 & -12 & 4.29 \\
\hline Posterior corpus callosum & $\mathrm{R}$ & & 12 & -33 & 21 & 3.89 \\
\hline \multicolumn{7}{|c|}{ Alternative model separating early and late maintenance phase } \\
\hline \multicolumn{7}{|c|}{ Memory load: early maintenance } \\
\hline Middle temporal gyrus & $\mathrm{R}$ & 21 & 48 & 12 & -33 & 4.57 \\
\hline Angular gyrus & $\mathrm{R}$ & 19 & 42 & -81 & 36 & 4.45 \\
\hline Precuneus & $\mathrm{R}$ & 7 & 9 & -63 & 45 & 4.36 \\
\hline Cerebellum & $\mathrm{R}$ & & 15 & -54 & -21 & 3.98 \\
\hline \multicolumn{7}{|l|}{ Memory load: late maintenance } \\
\hline Hippocampus & $\mathrm{L}$ & 28 & -24 & -21 & -15 & 4.10 \\
\hline
\end{tabular}

The table displays the coordinates of the maximally activated voxel in each activation cluster. L, Left; $R$, right; BA, Brodmann's area.

activity during a time period beginning several seconds after the presentation of the last stimulus (which could be either a face or a scrambled face) because the BOLD response during the first few seconds at the beginning of the delay period may still be contaminated with encoding-related activity (Zarahn et al., 1997; Postle et al., 2000). Therefore, to confirm that our findings reflect maintenance activity that is independent of encoding activity, we reestimated our data using a modified set of regressors, which separately modeled the first $4 \mathrm{~s}$ and the remaining 2-6 s of the maintenance phase. The results are shown in the supplemental Figure 4 (available at www.jneurosci.org as supplemental material). We found a significant memory load-dependent activation of the left hippocampus during the later part of the maintenance phase $\left(t_{(22)}=4.10\right.$; MNI coordinates, $\left.-24,-21,-15\right)$.

\section{Discussion}

\section{Neural processes in the MTL during WM maintenance}

Our iEEG recordings reported above revealed a positive shift of the medial temporal (rhinal and hippocampal) DC potential during maintenance of a single item and gradual negative shifts during maintenance of multiple items. A negative DC potential shift likely corresponds to increased firing and/or increased synaptic activation and membrane potential depolarization of large groups of neurons (Speckmann and Elger, 1999). This intracellular depolarization must be extended in time, for example by persistent firing and/or a persistent increase in synaptic input, to produce an extracellular DC shift (rather than a transient deflection). Such sustained alterations of neural activity likely reflect WM maintenance (Fuster and Alexander, 1971; Goldman-Rakic, 1995). The positive shift of the DC potential during maintenance of a single item (Figs. 3, 4, light gray traces) is therefore likely attributable to a sustained decrease in neural activity. However, the interpretation of this decrease relative to baseline is difficult as it depends on the exact definition of the baseline period. We thus suggest that these results should be taken very cautiously and need to be tested in future studies.

Synchronized activity in the gamma frequency range is a prominent EEG pattern during activated states of the MTL (Bragin et al., 1995; Chrobak and Buzsáki, 1996) that is related to cellular correlates of memory formation and WM maintenance (Herrmann et al., 2004; Axmacher et al., 2006). To address the issue of MTL gamma-band activity during WM maintenance, we performed time-frequency analyses and extracted frequency- 
a

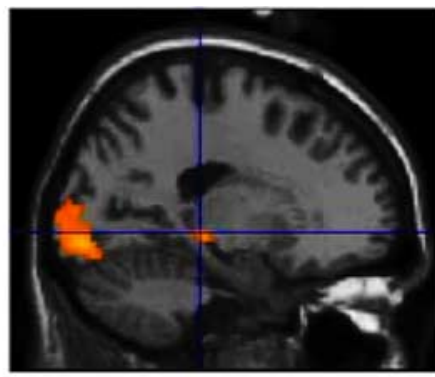

b

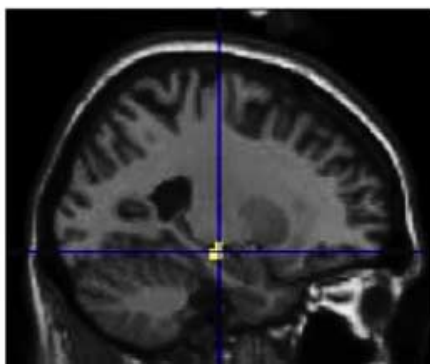

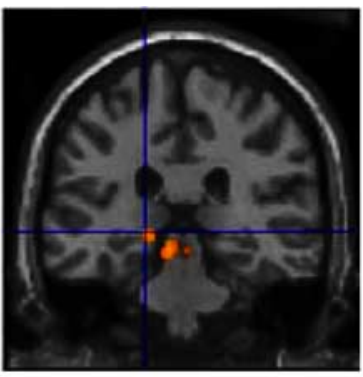
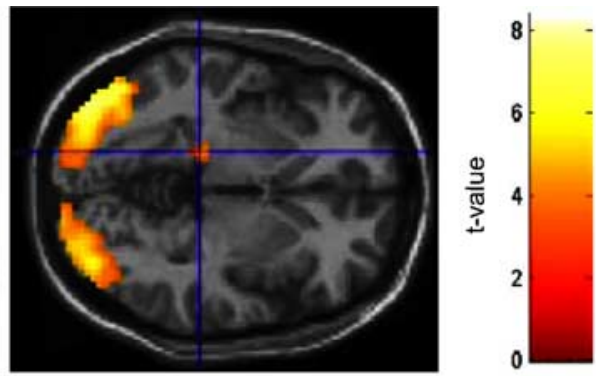

hippocampus
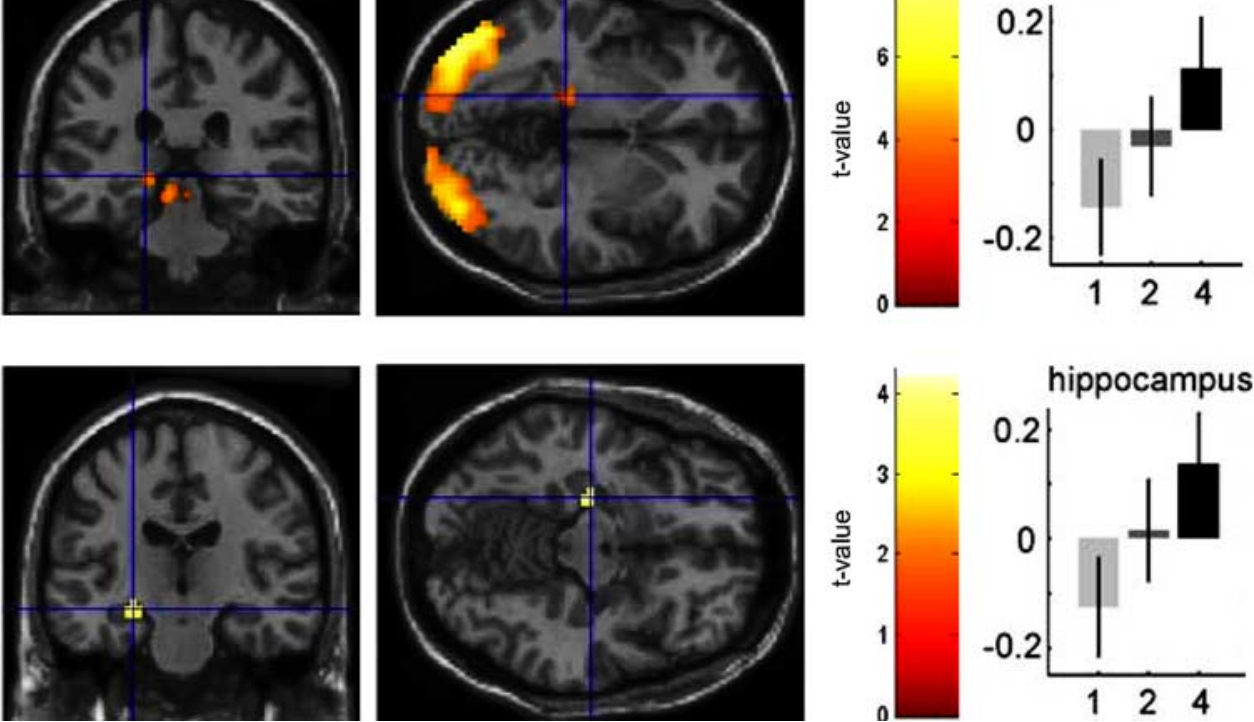

hippocampus

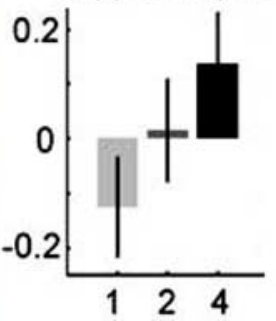

Figure 7. Memory load-dependent activation during processing of an increasing number of items during encoding and maintenance. $\boldsymbol{a}, \boldsymbol{b}$, Memory load-dependent hippocampal activation during encoding $(\boldsymbol{a})$ and maintenance $(\boldsymbol{b})$. The right column shows parameter estimates for contrast estimates of the most activated voxel in the left MTL in each condition (mean \pm SD).

specific power values reflecting local synchronization of neural activity (Klimesch, 1996). Our wavelet analyses of gamma-band activity revealed a decreased power during maintenance of a single item compared with baseline (Fig. 5, left column). Again, it should be noted that the decreased gamma power is only relative to gamma-band activity during baseline and, thus, should not be overstated; a more extended discussion of previous findings related to gamma-band activity is provided in the supplemental material (available at www.jneurosci.org).

We observed that with increasing memory load the positive DC potential shift was significantly reduced and even turned into a slightly negative shift when four items were encoded (Fig. 4) and maintained (Fig. 3). Again, the DC potential shifts persisted over several seconds. These findings correspond to a previous in vitro study (Egorov et al., 2002) indicating that stimulation of the entorhinal cortex induced sustained cellular activity that outlasted stimulation and, thus, may be a mechanism for stimulus maintenance during a delay period. Even more striking was the observation, in that study, of a gradual increase in firing rate and membrane depolarization for repeated stimulations, similar to the increasingly more negative DC potential shift during encoding of subsequent items reported in our study (Fig. 4). The increased gamma-band activity during maintenance of multiple items confirms predictions from an influential computer model by Jensen, Lisman and colleagues (Lisman and Idiart, 1995; Jensen and Lisman, 2005) that suggests that multiple items are encoded by subsequent gamma cycles during a theta cycle.

\section{fMRI data confirm increased MTL activation during multiple-item WM}

Consistent with the iEEG findings, our fMRI data revealed increased activation of the MTL during encoding and maintenance of multiple compared with single items. Furthermore, we found clear evidence for memory load-dependent activation of the MTL during both encoding and maintenance, arguing against the hypothesis that the changes of the DC potential and gamma-band activity within the MTL solely reflect unspecific activation of extensive brain regions. The apparent lack of MTL activation, or even deactivation, during maintenance of single items is consistent with a previous study by Piekema et al. (2006), but is in contrast to previous studies reporting increased MTL activity during WM delay periods even for single items (Ranganath and D'Esposito, 2001; Schon et al., 2004; Nichols et al., 2006). This divergence may be attributable to the baseline period (with respect to which activation or inactivation during specific trial periods are determined), which is fundamentally equivocal in fMRI experiments (Stark and Squire, 2001). Our paradigm comprised trials at which a different number of items has to be maintained, making it more demanding than experiments at which only single items have to be maintained. Furthermore, although the use of 30-s-long "break" periods as baseline (instead of intertrial intervals) has the advantage that any BOLD response related to stimulus presentation and maintenance has decayed, this "default mode" might involve considerable activation of a variety of different regions (Gusnard et al., 2001; Raichle et al., 2001). Thus, more "tonic" MTL activation may occur in our paradigm and contribute to the baseline than, for instance, in a simple delayed match to sample experiment in which the intertrial intervals served as a baseline. Together, we suggest that the apparent deactivation observed in our study strongly depends on the specific baseline definition and should thus await additional confirmation in future studies.

In our fMRI data, we observed memory load effects in the prefrontal cortex during the encoding phase, but not during the delay period. Although it might be argued that this is in apparent discrepancy to a wide range of literature demonstrating the importance of the prefrontal cortex for WM (Fuster and Alexander, 1971; Kubota and Niki, 1971; Cohen et al., 1997; Courtney et al., 1997), most of these studies used highly familiar stimuli such as words instead of trial-unique novel stimuli. In fact, several previous studies found a dissociation between the neural correlates of WM for familiar items in the frontal lobe and for novel items in the hippocampus (Stern et al., 2001; Ranganath and D'Esposito, 2001; Zarahn et al., 2005). We thus suppose that the absence of prefrontal activation during the delay period might be attributable to the stimulus material used. Conversely, the MTL might be 
more important than the prefrontal cortex for the short-term maintenance of complex novel items. This would imply that the differential contribution of the prefrontal cortex and MTL to memory processes depends on the stimulus type rather than the length of the retention interval.

\section{Cognitive processes engaged during the Sternberg task}

Exactly which processes in the MTL are measured by this experiment? Does the increased activity during maintenance of multiple items indeed reflect WM maintenance, or is it rather caused by the enhanced recruitment of LTM processes? Previous studies indeed showed that activation of the MTL during item maintenance predicts subsequent LTM for these items (Schon et al., 2004; Ranganath et al., 2005). Only WM for single items was tested in these studies, however. We argue for three reasons that it is unlikely that our results solely reflect LTM processes. First, load-dependent shifts of the DC potential depended on correct responses in the WM experiment (supplemental Table 2, available at www.jneurosci.org as supplemental material), but were independent of successful retrieval in a subsequent LTM recognition task (supplemental Results, supplemental Fig. 2, available at www.jneurosci.org as supplemental material). Second, we did not observe a load effect on LTM recognition in the behavioral study, suggesting that faces from trials with a different number of items were encoded equally likely. However, one principal caveat of the subsequent long-term recognition task was the poor performance because of the large number of items tested. We cannot rule out that this poor performance was caused by interference during consolidation and retrieval rather than a lack of LTM encoding. However, the large number of faces in the Sternberg task was required to investigate WM of trial-unique items. Third, the DC shifts measured with iEEG clearly reflect sustained changes in neural activity different from the more transient patterns observed in a subsequent memory paradigm, in particular within the rhinal cortex (Fernández et al., 1999; Fell et al., 2001). Although, it should be noted that a direct comparison of these two experiments is difficult because of the use of different stimulus material, different presentation times, etc.

Our task did not explicitly require that relations between items or sequences of items were processed. Thus, additional studies are needed to address the question whether the MTL specifically supports maintenance of relations (Cohen and Eichenbaum, 1993; Eichenbaum, 2004) or sequences (Eichenbaum et al., 1999; Henke et al., 1999; Schendan et al., 2003; Jensen and Lisman, 2005; Kumaran and Maguire, 2006), or whether it is similarly engaged in all memory processes involving multiple items.

To summarize, our data show an incremental enhancement of neural activity and BOLD response within the human MTL with increasing WM load. These findings argue against the traditional view that the MTL does not contribute to WM. Furthermore, the deactivation during maintenance of one item and activation during maintenance of multiple items suggests that the neural processes in the MTL depend crucially on the number of items kept in WM.

\section{References}

Aggleton JP, Shaw C, Gaffan EA (1992) The performance of postencephalitic amnesic subjects on two behavioural tests of memory: concurrent discrimination learning and delayed matching-to-sample. Cortex 28:359-372.

Axmacher N, Mormann F, Fernandez G, Elger CE, Fell J (2006) Memory formation by neuronal synchronization. Brain Res Rev 52:170-182.

Bragin A, Jando G, Nadasdy Z, Hetke J, Wise K, Buzsáki G (1995) Gamma
$(40-100 \mathrm{~Hz})$ oscillation in the hippocampus of the behaving rat. J Neurosci 15:47-60.

Brett M, Anton JL, Valabregue R, Poline JP (2002) Region of interest analysis using an SPM toolbox. Abstract presented at the Eighth International Conference on Functional Mapping of the Human Brain, Sendai, Japan, June.

Cave CB, Squire LR (1992) Intact verbal and nonverbal short-term memory following damage to the human hippocampus. Hippocampus 2:151-163.

Chrobak JJ, Buzsáki G (1996) High-frequency oscillations in the output networks of the hippocampal-entorhinal axis of the freely behaving rat. J Neurosci 16:3056-3066.

Cohen JD, Perlstein WM, Braver TS, Nystrom LE, Noll DC, Jonides J, Smith EE (1997) Temporal dynamics of brain activation during a WM task. Nature 386:604-608.

Cohen NJ, Eichenbaum H (1993) Memory, amnesia, and the hippocampal system. Cambridge, MA: MIT.

Courtney SM, Ungerleider LG, Keil K, Haxby JV (1997) Transient and sustained activity in a distributed neural system for human WM. Nature 386:608-611.

Daubechies I (1990) The wavelet transform, time-frequency localization and signal analysis. IEEE Trans Inform Theory 36:961-1005.

Delorme A, Makeig S (2004) EEGLAB: an open source toolbox for analysis of single-trial EEG dynamics, J Neurosci Methods 134:9-21.

Egorov AV, Hamam BN, Fransen E, Hasselmo ME, Alonso AA (2002) Graded persistent activity in entorhinal cortex neurons. Nature 420:173-178.

Eichenbaum H (2004) Hippocampus: cognitive processes and neural representations that underlie declarative memory. Neuron 44:109-120.

Eichenbaum H, Dudchenko P, Wood E, Shapiro M, Tanila H (1999) The hippocampus, memory, and place cells: is it spatial memory or a memory space? Neuron 23:209-226.

Fell J, Klaver P, Lehnertz K, Grunwald T, Schaller C, Elger CE, Fernández G (2001) Human memory formation is accompanied by rhinalhippocampal coupling and decoupling. Nat Neurosci 4:1259-1264.

Fernández G, Effern A, Grunwald T, Pezer N, Lehnertz K, Dumpelmann M, Van Roost D, Elger CE (1999) Real-time tracking of memory formation in the human rhinal cortex and hippocampus. Science 285:1582-1585.

Fuster JM, Alexander GE (1971) Neuron activity related to short-term memory. Science 173:652-654.

Goldman-Rakic PS (1995) Cellular basis of working memory. Neuron 14:477-485.

Gusnard DA, Raichle ME, Raichle ME (2001) Searching for a baseline: functional imaging and the resting human brain. Nat Rev Neurosci 2:685-694.

Hannula DE, Tranel D, Cohen NJ (2006) The long and the short of it: relational memory impairments in amnesia, even at short lags. J Neurosci 262:8352-8359.

Henke K, Weber B, Kneifel S, Wieser HG, Buck A (1999) Human hippocampus associates information in memory. Proc Natl Acad Sci USA 96:5884-5889.

Herrmann CS, Munk MH, Engel AK (2004) Cognitive functions of gammaband activity: memory match and utilization. Trends Cogn Sci 8:347-355.

Huynh H, Feldt LS (1976) Estimation of the box correction for degrees of freedom from sample data in the randomized plot and split plot designs. J Educ Stat 1:69-82.

Jensen O, Lisman JE (2005) Hippocampal sequence-encoding driven by a cortical multi-item WM buffer. Trends Neurosci 28:67-72.

Klimesch W (1996) Memory processes, brain oscillations and EEG synchronization. Int J Psychophysiol 24:61-100.

Kubota K, Niki H (1971) Prefrontal cortical unit activity and delayed alternation performance in monkeys. J Neurophysiol 34:337-347.

Kumaran D, Maguire EA (2006) The dynamics of hippocampal activation during encoding of overlapping sequences. Neuron 49:617-629.

Lisman JE, Idiart MA (1995) Storage of $7 \pm 2$ short-term memories in oscillatory subcycles. Science 267:1512-1515.

Nichols EA, Kao YC, Verfaellie M, Gabrieli JD (2006) Hippocampus, working memory and long-term memory for faces: evidence from fMRI and global amnesia for involvement of the medial temporal lobes. Hippocampus 16:604-616.

Olson IR, Page K, Moore KS, Chatterjee A, Verfaellie M (2006) Working memory for conjunctions relies on the medial temporal lobe. J Neurosci 26:4596-4601. 
Penfield W, Milner B (1958) Memory deficit produced by bilateral lesions in the hippocampal zone. AMA Arch Neurol Psychiatry 79:475-497.

Piekema C, Kessels RP, Mars RB, Petersson KM, Fernandez G (2006) The right hippocampus participates in short-term memory maintenance of object-location associations. NeuroImage 33:374-382.

Postle BR, Zarahn E, D’Esposito M (2000) Using event-related fMRI to assess delay-period activity during performance of spatial and nonspatial WM tasks. Brain Res Brain Res Protoc 5:57-66.

Raichle ME, MacLeod AM, Snyder AZ, Powers WJ, Gusnard DA, Shulman GL (2001) A default mode of brain function. Proc Natl Acad Sci USA 98:676-682.

Ranganath C, Blumenfeld RS (2005) Doubts about double dissociations between short- and long-term memory. Trends Cogn Sci 9:374-380.

Ranganath C, D'Esposito M (2001) Medial temporal lobe activity associated with active maintenance of novel information. Neuron 31:865-873.

Ranganath C, Cohen MX, Brozinsky CJ (2005) Working memory maintenance contributes to long-term memory formation: neural and behavioral evidence. J Cogn Neurosci 17:994-1010.

Schendan HE, Searl MM, Melrose RJ, Stern CE (2003) An FMRI study of the role of the medial temporal lobe in implicit and explicit sequence learning. Neuron 37:1013-1025.

Schon K, Hasselmo ME, Lopresti ML, Tricarico MD, Stern CE (2004) Persistence of parahippocampal representation in the absence of stimulus input enhances long-term encoding: a functional magnetic resonance imaging study of subsequent memory after a delayed match-to-sample task. J Neurosci 24:11088-11097.

Scoville WB, Milner B (1957) Loss of recent memory after bilateral hippocampal lesions. J Neurol Neurosurg Psychiatry 20:11-21.
Speckmann EJ, Elger CE (1999) Introduction to the neurophysiological basis of the EEG and DC potentials. In: Electroencephalography, Ed 4 (Niedermeyer E, Lopes da Silva F, eds), pp 15-27. Baltimore: Lippincott, Williams and Wilkins.

Stark CE, Squire LR (2001) When zero is not zero: the problem of ambiguous baseline conditions in fMRI. Proc Natl Acad Sci USA 98:12760-12766.

Stern CE, Sherman SJ, Kirchhoff BA, Hasselmo ME (2001) Medial temporal and prefrontal contributions to working memory tasks with novel and familiar stimuli. Hippocampus 11:337-346.

Sternberg S (1975) Memory scanning: new findings and current controversies. Q J Exp Psychol 18:643-662.

Suzuki WA, Miller EK, Desimone R (1997) Object and place memory in the macaque entorhinal cortex. J Neurophysiol 78:1062-1081.

Torrence C, Compo GP (1998) A practical guide to wavelet analysis. B Am Meteorol Soc 79:61-78.

Van Roost D, Solymosi L, Schramm J, Van Oosterwyck B, Elger CE (1998) Depth electrode implantation in the length axis of the hippocampus for the presurgical evaluation of medial temporal lobe epilepsy: A computed tomography-based stereotactic insertion technique and its accuracy. Neurosurgery 43:819-826.

Young BJ, Otto T, Fox GD, Eichenbaum H (1997) Memory representation within the parahippocampal region. J Neurosci 17:5183-5195.

Zarahn E, Aguirre G, D’Esposito M (1997) A trial-based experimental design for fMRI. NeuroImage 6:122-138.

Zarahn E, Rakitin B, Abela D, Flynn J, Stern Y (2005) Positive evidence against human hippocampal involvement in WM maintenance of familiar stimuli. Cereb Cortex 15:303-316. 